\title{
A NOTE ON WELL DISTRIBUTED SEQUENCES
}

\section{B. LAWTON}

1. Let $\{x\}$ denote the fractional part of the real number $x$ and let $I=I(a, b)$ denote the closed interval $[a, b]$. We suppose throughout that $0 \leqq a<b \leqq 1$. Then a sequence $s_{n}$ of real numbers is said to be well distributed mod 1 if, and only if, for every $I$,

$$
\lim _{t \rightarrow \infty} t^{-1} \sum_{k=n+1 ;\left\{S_{k}\right\} \in I}^{n+t} 1=b-a,
$$

holds uniformly in $n$. This definition was introduced by Petersen [3] and evidently represents a greater restriction on a sequence than the classical uniform distribution condition:

$$
\lim _{t \rightarrow \infty} t^{-1} \sum_{k=1 ;\left\{S_{k}\right\} \in I}^{t} 1=b-a, \text { for every } I .
$$

Nevertheless, certain theorems on uniformly distributed sequences can be formulated in terms of the new definition. For example, Petersen [3, Theorems 2, 3] has shown that Weyl's criterion [2, Satz 6] would take the modified form: a sequence $s_{n}$ is well distributed mod 1 if, and only if, for each integer $h>0$,

$$
\lim _{t \rightarrow \infty} t^{-1} \sum_{k=n+1}^{n+t} e\left(h s_{k}\right)=0, \text { uniformly in } n,
$$

where $e(\theta)$ denotes exp $(2 \pi i \theta)$. In this note, I give two further examples:

Theorem 1. A sequence $s_{n}$ is well distributed $\bmod 1$ if, for each integer $m>0$, the sequence $s_{n+m}-s_{n}$ is well distributed mod 1.

Theorem 2. Suppose that the polynomial

$$
s_{n}=\alpha_{r} n^{r}+\alpha_{r-1} n^{r-1}+\cdots+\alpha_{0}
$$

has at least one irrational coefficient $\alpha_{j}$ with $j>0$. Then the sequence $s_{n}$ $(n=1,2, \cdots)$ is well distributed $\bmod 1$.

We follow the classical treatment as given, for example, in Cassel's Tract [1], but since Theorem 2 requires only routine changes (as a direct consequence of Theorem 1), we omit details. For Theorem 1, an essential link in the relative behaviour of the sequences $s_{n}$ and

Received by the editors, March 5, 1959. 
$s_{n+m}-s_{n}$ is provided by the inequality (for a proof, see e.g., [1, Lemma 3]):

$$
\begin{aligned}
H^{2}\left|\sum_{q=1}^{Q} u_{q}\right|^{2} \leqq & H(H+Q-1) \sum_{q=1}^{Q}\left|u_{q}\right|^{2} \\
& +2(H+Q-1) \sum_{h=1}^{H-1}(H-h)\left|\sum_{q=1}^{Q-h} \bar{u}_{q} u_{q+h}\right|,
\end{aligned}
$$

where $H, Q$ are arbitrary positive integers with $H \leqq Q$ and $u_{1}, \cdots, u_{Q}$ denote any complex numbers. With our notation, where

$$
\tau=H, t=Q, u_{1}=e\left(h s_{n+1}\right), \cdots, u_{t}=e\left(h s_{n+t}\right)
$$

this becomes

(5) $\tau^{2}\left|\sum_{k=n+1}^{n+t} e\left(h s_{k}\right)\right|^{2} \leqq \tau(\tau+t-1) t$

$$
+2(\tau+t-1) \sum_{j=1}^{\tau-1}(\tau-j)\left|\sum_{k=n+1}^{n+t-j} e\left(h s_{k+j}-h s_{k}\right)\right|
$$

and is, in fact, directly applicable to our problem in the weaker form

$$
\begin{aligned}
\left|\sum_{k=n+1}^{n+t} e\left(h s_{k}\right)\right|^{2} & \\
& \leqq 2 t^{2} \tau^{-1}+4 t^{2} \tau^{-1} \sum_{j=1}^{\tau-1}(t-j)^{-1}\left|\sum_{k=n+1}^{n+t-j} e\left(h s_{k+j}-h s_{k}\right)\right| .
\end{aligned}
$$

2. Proof of Theorem 1. Let $\epsilon>0$ and suppose that, for each positive integer $m$, the sequence $s_{n+m}-s_{n}$ is well distributed mod 1 . Then, by (3), there corresponds to each $\epsilon>0$ an integer $T=T(\epsilon, h, m)$, independent of $n$, such that

$$
\left|t^{-1} \sum_{k=n+1}^{n+t} e\left(h s_{m+k}-h s_{k}\right)\right|<\epsilon, \quad \text { for all } t>T .
$$

Consider now the terms on the right of (6), with $\tau=\left[\epsilon^{-1}\right]+2$. Then for all $t>T+\tau$, we have

$$
4 t^{2} \tau^{-1} \sum_{j=1}^{\tau-1}(t-j)^{-1}\left|\sum_{k=n+1}^{n+t-j} e\left(h s_{k+j}-h s_{k}\right)\right|<4 t^{2} \tau^{-1}(\tau-1) \epsilon,
$$

since $t-j>T+\tau-j>T$. Hence

$$
t^{-2}\left|\sum_{k=n+1}^{n+t} e\left(h s_{k}\right)\right|^{2}<(2 \epsilon+4 \epsilon)=6 \epsilon
$$


for all $t>T+\tau$. Since $T+\tau$ is independent of $n$ it follows, by (3), that $s_{n}$ is well distributed mod 1 .

\section{REFERENCES}

1. J. W. S. Cassels, An introduction to diophantine approximation, Cambridge University Press, 1959, Chapter IV, §5.

2. J. F. Koksma, Diophantische Approximationen, Ergebnisse der Mathematik, vol. IV, Berlin, Springer, 1937, Chapter VIII, \$3.

3. G. M. Petersen, Almost convergence and uniformly distributed sequences, Quart. J. Math. vol. 7 (1956) pp. 188-191.

4. H. Weyl, Über die Gleichverteilung von Zahlen mod Eins, Math. Ann. vol. 77 (1916) pp. 313-352.

BedFord College, London

\section{THE EXISTENCE OF NONABSOLUTELY CONVERGENT FOURIER SERIES ON COMPACT GROUPS}

GUSTAVE RABSON

1. I. E. Segal has proved [1] that on a locally compact abelian group with infinitely many elements the class of complex-valued functions which are absolutely convergent Fourier transforms is a dense set of the first category in the space of complex-valued continuous functions vanishing at infinity. Although he assumes throughout that the group is abelian, his Lemma 5, which states the result for compact metric abelian groups, applies as well without assuming the group to be abelian with only minor changes in the wording of the proof. The assumption that the group is metric can then be eliminated by using the fact that any compact infinite group has a compact infinite second countable factor group (which is easy to prove using the fact that there are unitary representations separating every pair of points) and then proceeding as we do in the final steps of the proof of our main theorem.

In this paper we shall prove that on every compact group, not of dimension zero, there is a continuous complex-valued function which is not an absolutely convergent Fourier transform. This theorem is weaker than Segal's since the fact that the group is not of dimension zero seems to be intrinsic in the proof and we conclude nothing about the category of the set of all absolutely convergent Fourier transforms. However, the proof being entirely different from Segal's, and being constructive, may be of some interest. The author is indebted

Received by the editors September 24, 1958 and, in revised form, March 5, 1959. 